\title{
Scattering of Light on Droplets and Spherical Objects: 100 Years of Mie Scattering
}

\author{
Maciej Kolwas \\ Institute of Physics, Polish Academy of Sciences, \\ Al. Lotników 32/46, 02-668 Warszawa, Poland \\ e-mail: Kolwas@ifpan.edu.pl
}

(Received: 29 June 2010; accepted: 13 September 2010)

\begin{abstract}
In 1908 Gustav Mie published paper about scattering of light on spherical objects. This paper based on classical electrodynamics (Maxwell equations) is one of the most cited physical papers. In this paper on the occasion of 100 anniversary of Mie scattering several contemporary applications of Mie theory are shown, starting from light scattering on small metal droplets, until investigation of speed of evaporation of water micro droplets with and without nano inclusions.
\end{abstract}

Key words: Mie scattering, plasmons, water drop evaporation

\section{MIE THEORY}

The scattering of light on spherical objects is a phenomenon which can be observed everywhere. For example, looking at a sunset at the seacoast (Fig. 1) one can remark colourful clouds - scattering of different wavelengths of light on diverse in dimension but spherical water droplets composing clouds. The blue colour of sky as well as the yellow and reddish colours of sunset are another example of the scattering phenomena - a selection of colours by scattering cross section dependence on wavelength and radius of scatterers.

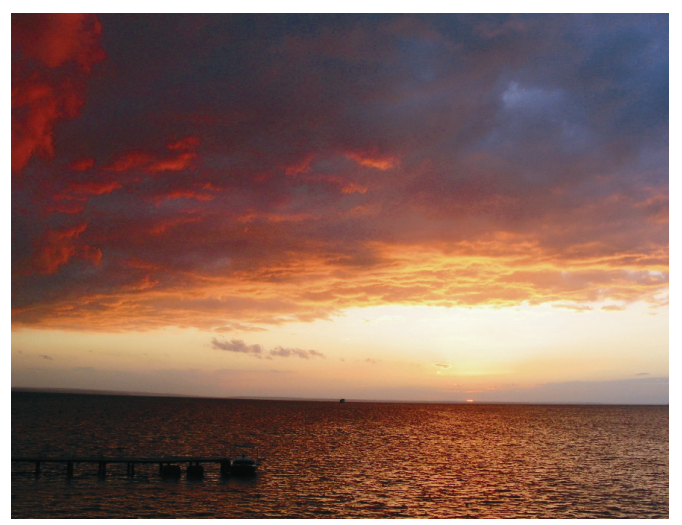

Fig. 1. Sunset at the Baltic see cost near Gdańsk
The optical phenomena seen in the fog, mist as well as city smog are mainly due to scattering by spherical micro objects, especially in wet atmosphere. Very well known is the influence of dust - sand grains from a desert and soot from the combustion on the composition and creation of clouds, therefore monsoons and generally speaking Earth weather.

But in the origin of Mie theory present is different observation than scattering of light on small atmospheric objects. Michel Faraday wrote in [1]: "Colloids of gold particles, even the mere variation in the size of particles gives rise to a variety of colours".

Despite the extremely low concentration of silver particles $(<10-2$ weight $\%)$, suspensions show bright colours, which depend on the size of particles especially in the range particle radius of the order $10 \mathrm{~nm}$ - few hundreds of $\mathrm{nm}$. The difference in colours viewed in transmitted light and when a light is shone from the front side shows that the observed colours for given particle size are not a simple compliment of the absorbed ones.

In 1908, Gustav Mie, a German physicist working at that time in Greifswald, published a famous theoretical paper entitled "Contributions to the optics of turbid media, particularly colloidal metal suspension" and published in Annalen der Physik [2]. The description of light scattering on spherical objects was studied by Lorenz and Debye as 

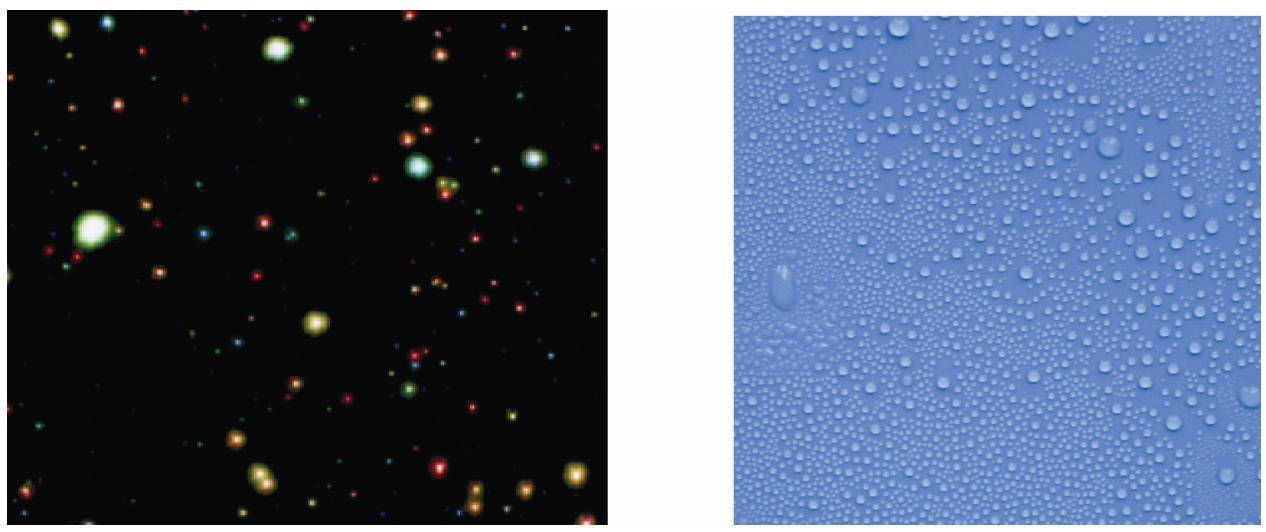

Fig 2. Scattering of white light by $30 \mathrm{~nm}$ silver nanoparticles and by clusters of nanoparticles of different size and shape (left). Scattering of light on water droplets does not change the colour of light with changing droplet dimension (right)

well, but Gustav Mie published his results in a very good journal, which is why today this approximation is mainly called the Mie theory [3].

The Mie theory is the solution of the Maxwell equation [4] with boundary conditions (continuity of electric and magnetic fields) on the surface of the particle, as schematically represented in Fig. 3. The particle is represented by a blue circle, and the electromagnetic field by water waves. The solutions were found with assumptions: a plane electromagnetic wave (representing light) illuminates a homogeneous, perfectly spherical particle embedded in an infinite homogenous host medium. The particle and the host medium can be absorbing and is possible to use complex refractive indices for both.

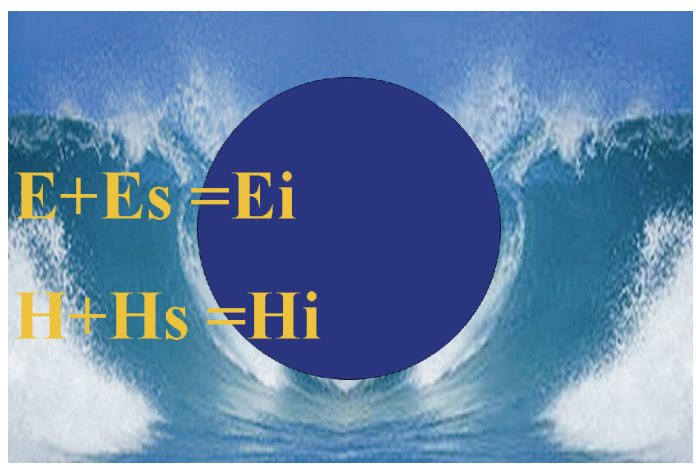

Fig. 3. Schematic representation of the Mie theory

The incident, internal, and scattered fields are represented as infinite series of terms consisting of functions of angular and radial variables. Therefore, the symmetry of object is transferred to the symmetry of solutions. The total field in the presence of the object - spherical particle or droplet - is represented as a vector sum of the incident and scattered fields.
As a solution, the angle distribution of scattered light as well as cross sections for extinction, scattering and absorption can be calculated.

In Fig. 4, cross sections efficiency $\sigma / \pi R^{2}$ are shown for clean water (red) and dusty (absorbing light) water.

The extinction of light is due to scattering only for clean water, while for dusty water absorption appears.

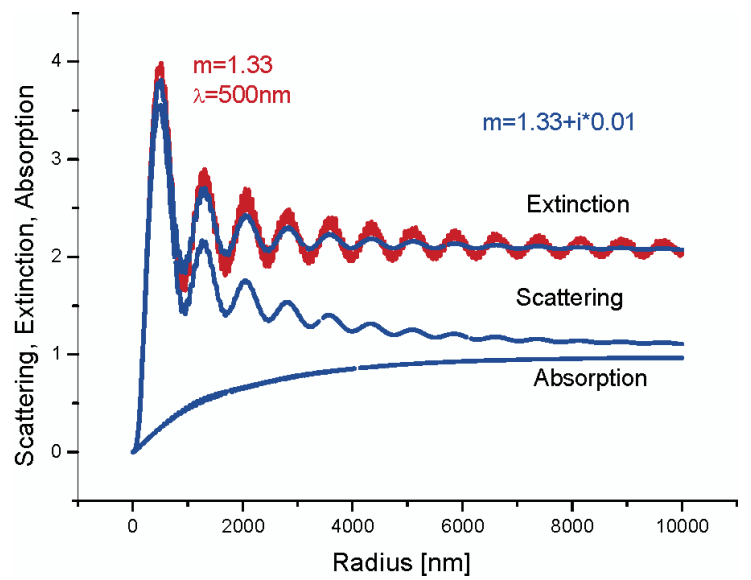

Fig. 4. Cross sections

Very interesting is a comparison of scattering on different materials (with different refractive indices).

Figure 5 shows the intensity of back scattered light $(633 \mathrm{~nm})$ for a growing particle of soot (strongly absorbing, almost like a black body), sodium and pure water. Until ca. $100 \mathrm{~nm}$ the object scatters proportionally to a square of volume, than after the almost "phase transition" the main tendency is the dependence on square of radius, i.e. surface of scatterer. But clear oscillations can be remarked - very dense for water and decaying with grows of a particle for the soot. Similarly, the scattering on metal (sodium) drop shows oscillations for radiuses bigger than $100 \mathrm{~nm}$. 

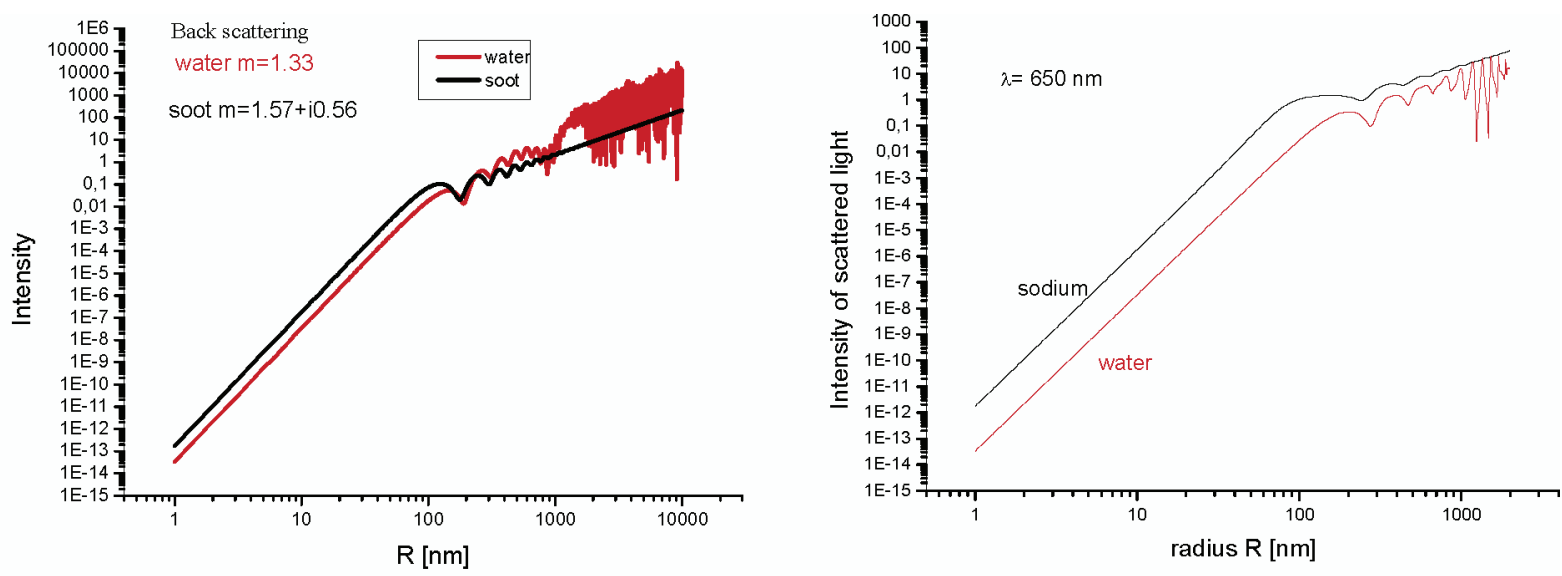

Fig. 5. Intensity of scattered light in the function of diameter of particle for water, soot and sodium
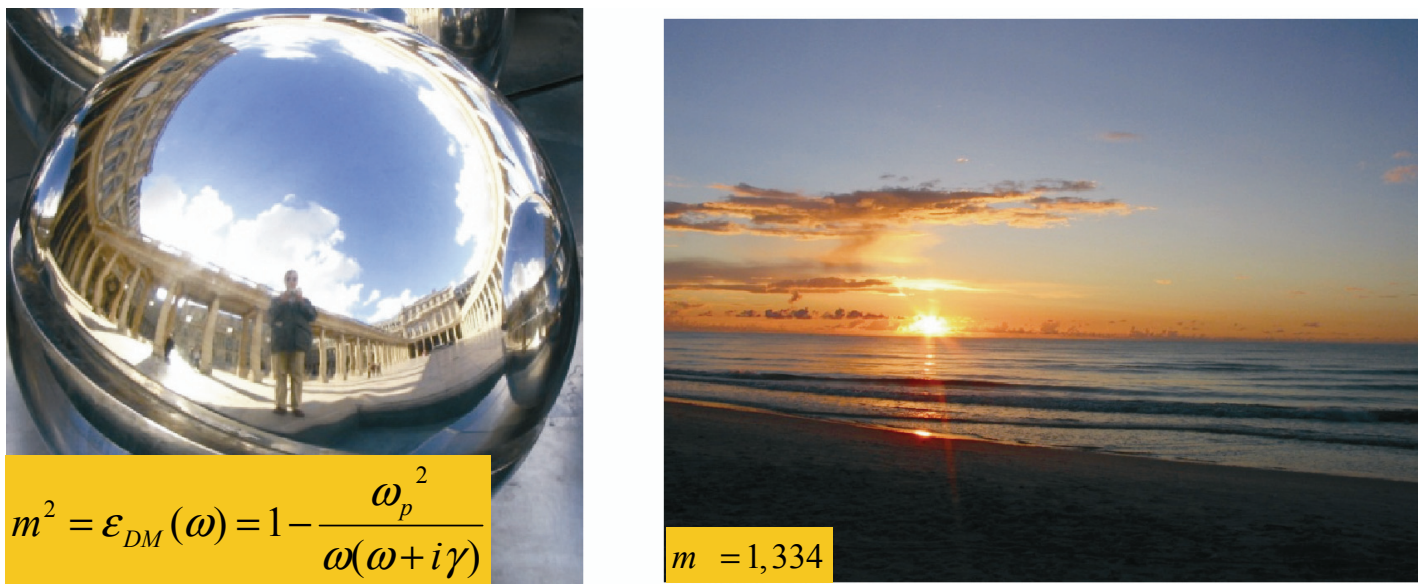

Fig. 6. Reflection on a metal sphere with the Drude type of dielectric constant and scattering on water droplets can be described using the Mie theory

To summarize - in the Mie theory scattered light depends on the radius of the scattering sphere (particle), refractive indices of the sphere and embedding medium, wavelength (colour) \& polarization of scattered light. The excellent agreement between Mie's theoretical results and the experiments demonstrate that the optical constants obtained for bulk samples could be used for particles as small as one hundredth of the wavelength.

\section{PLASMON RESONANCES IN SODIUM DROPLETS (CONDENSATION)}

The contemporary use of the Mie theory can be illustrated by two applications: a study of plasmon resonances in growing sodium clusters and an investigation of evaporating droplets of pure water and water with inclusions.
At first let us discuss shortly the evolution of scattered light intensity due to growth of sodium nanodroplets.

The evolution of right angle scattered light intensities was performed in two geometries (Fig. 8) [5-7].

Measured intensities of scattered light are presented in Fig. 9.

Looking at scattering of e.g. blue $457.9 \mathrm{~nm}$ laser light one can remark that a maximum of scattering for vertical polarization appears quicker, therefore for smaller clusters than maximum of scattering on $H$ polarization. This is due to the fact that on $V$ polarization the dipole component can participate in scattering while on $H$ polarization the smallest component is a quadrupole one. Theoretical values of plasmon resonances frequencies $\omega_{l}(R)$ are presented in Fig. 10a, together with radiative damping rates $\omega_{l}^{\prime \prime}(R)$ (Fig. 10b).

Plasmon resonances frequencies $\omega_{l}(R)$ found from experimental data (Fig. 9) are presented in Fig. 11. 

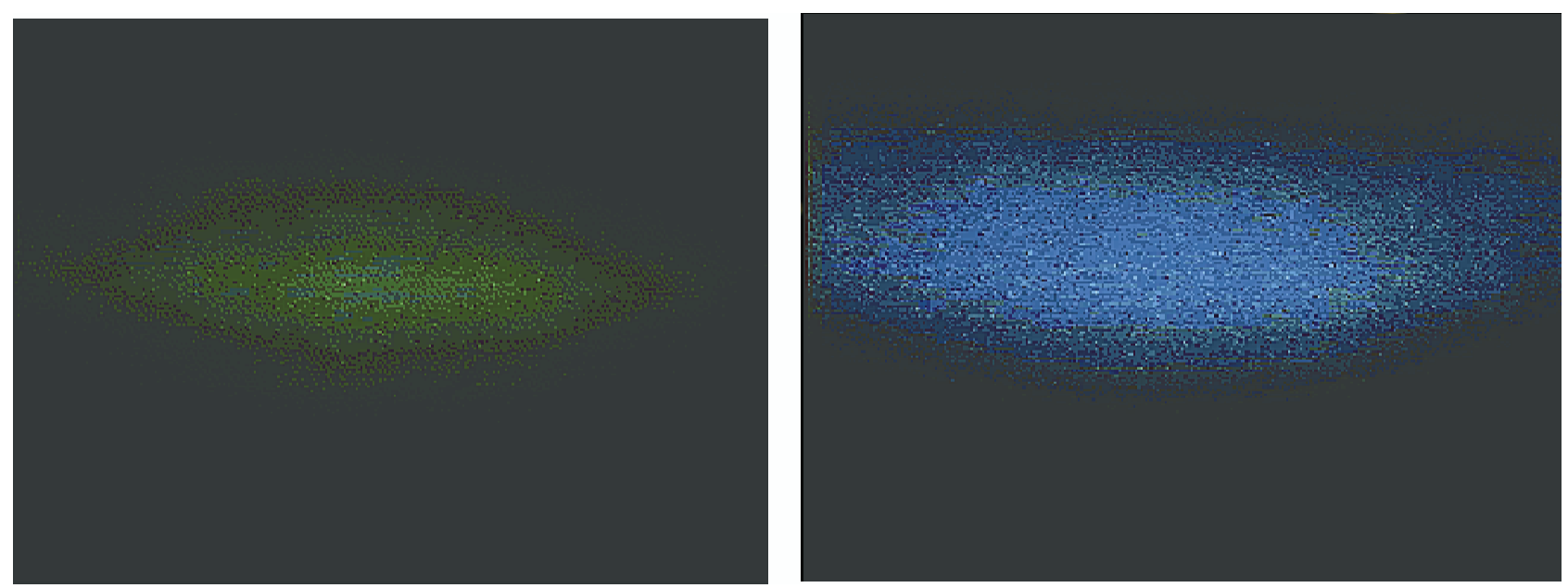

Fig. 7. Fluorescence of sodium dimers $\mathrm{Na}_{2}$ excited with blue $488 \mathrm{~nm}$ laser light (left). A few seconds after stimulation of condensation of sodium nanodroplets the scattering becomes elastic and colour change on laser one - blue

a)

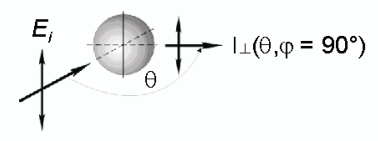

b)

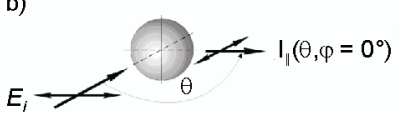

Fig. 8. Vertical $V($ a) and horizontal $H($ b) geometry of experiment

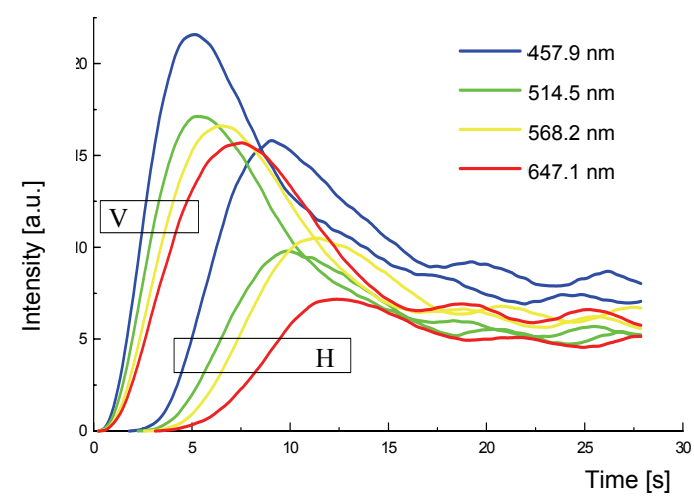

Fig. 9. Time evolution of intensity of scattered light on sodium nanodroplets for two geometries presented in Fig. 1 for four laser colours. The growth (nucleation) of clusters (droplets) was stimulated with laser at $t=0$

b)

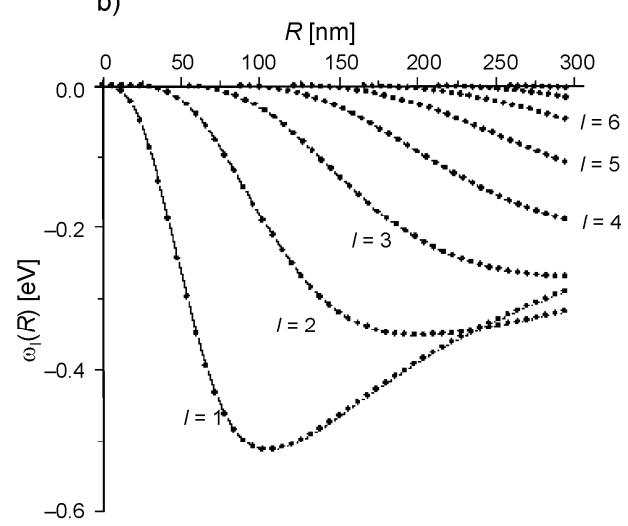

Fig. 10. Plasmon oscillations frequencies $\omega_{l}(R)$ and radiative damping rates $\omega_{l}^{\prime \prime}(R)$ in the function of the radius of particle (droplet) 


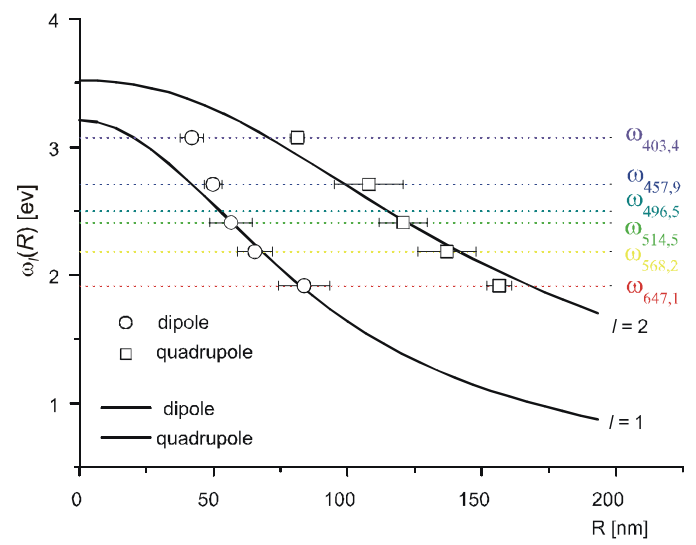

Fig. 11. Experimental (points and theoretical (lines ) values of dipole and quadrupole plasmon resonances [5-7]

\section{EVAPORATION OF WATER MICRO-DROPLETS}

To study evaporation of a single water droplet we had constructed an electro dynamical trap (Pauli trap) [8], kept in a small climatic chamber. A photo of model of trap (four rings electrodes) with scattered green laser light is shown in Fig. 13.

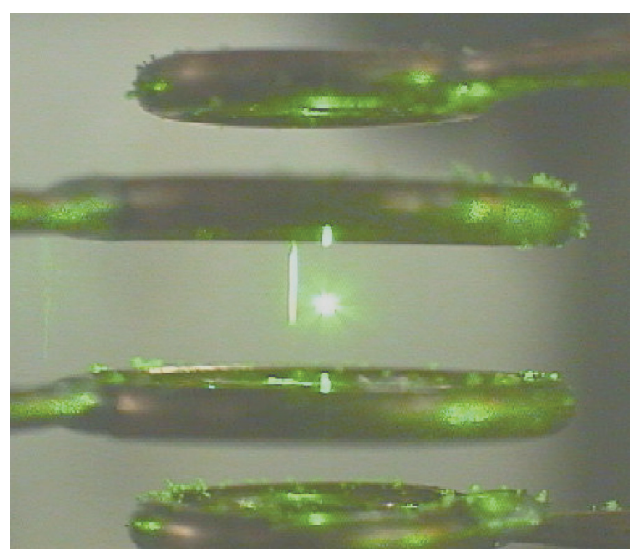

Fig. 12. Scattering of green light on trapped particles

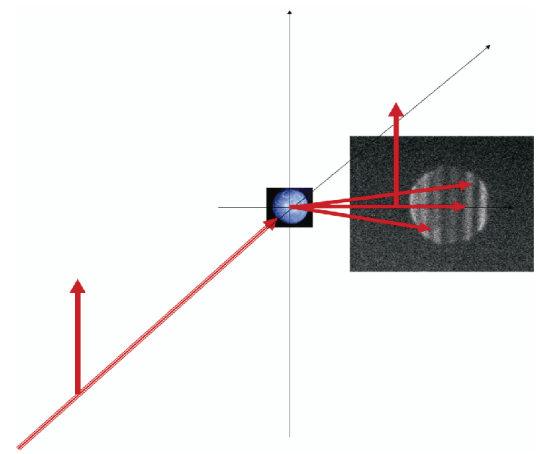

Fig. 13. Geometry of experiment
As a result of the experiment one obtains the picture of an interference pattern. The radius of a droplet can be found using the Mie theory from frequency of interference fringes.

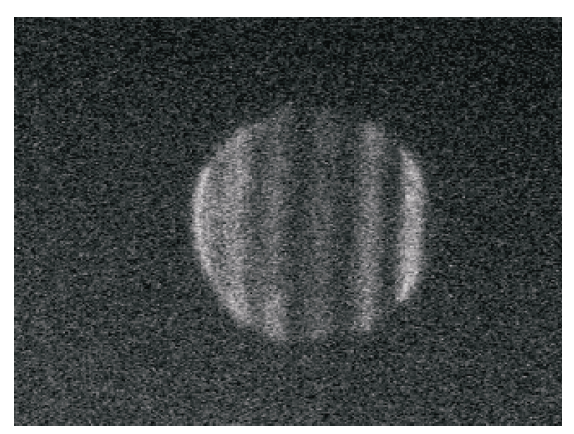

Fig. 14. Interference pattern seen at the right angle

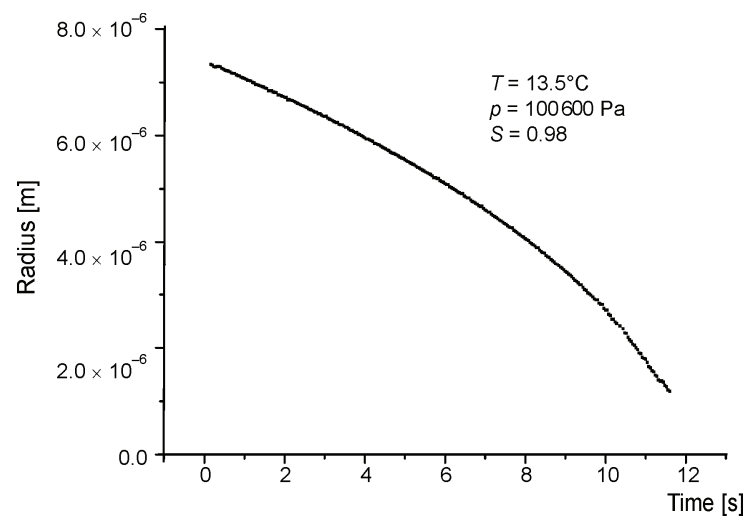

Fig. 15. Time evolution of droplet radius

From the analysis of time evolution of radius two parameters describing speed of evaporation of water from droplet surface can be found: so-called evaporation (condensation) or mass accommodation coefficient $\alpha$ and a thermal accommodation coefficient $\alpha_{T}$ [9].

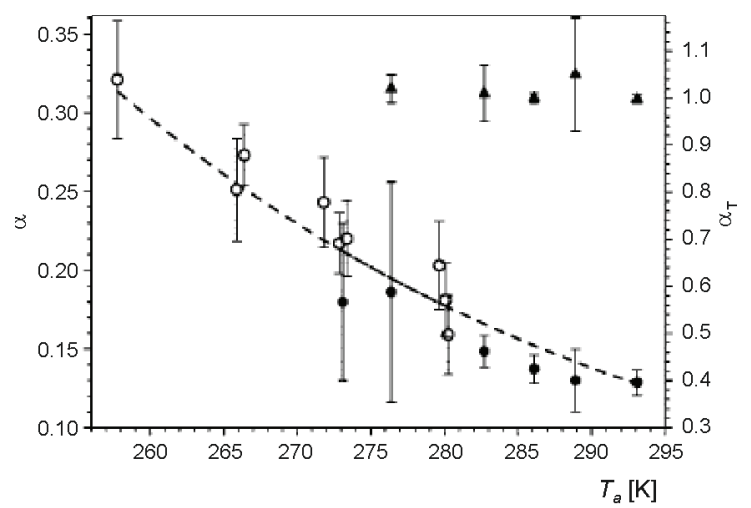

Fig. 16. Experimental results (dots) together with results from [10] 


\section{EVAPORATION OF WATER MICRO-DROPLETS OF NANOSPHERE SUSPENSION $(100 \mathrm{~nm})$}

The scheme of the experimental setup representing the electrodynamic trap and two counter propagating laser beams of orthogonal polarizations is shown in Fig. 17.

Green and red fringes/speckles in the detection channel arise from the coherent light scattering by the trapped droplet/crystallite into the solid angle of $\Delta \Theta=0.1 \mathrm{sr}$ around the right angle.
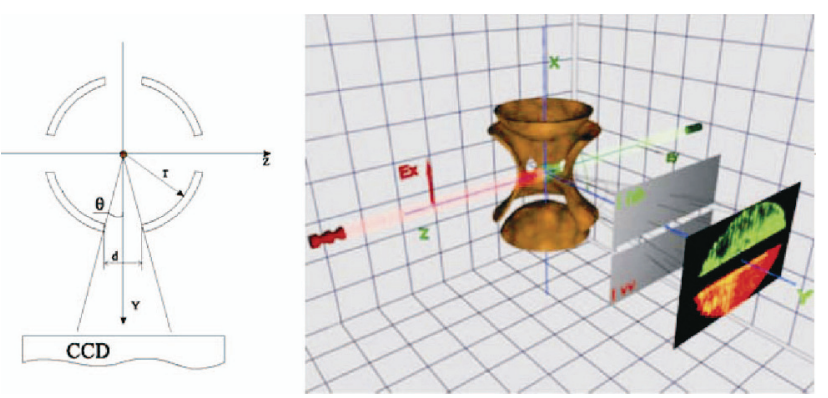

Fig. 17. The geometry of experiment. Polarisers standing in scattered light had orthogonal polarization to stop subsequently red and green scattered light

The droplet radius temporal dependence $a(t)$ (Fig. 18) was derived from the analysis of the frequency of the spatial modulation of the scattered light intensity. Using a theoretical description of radius evolution one can find the surface tension of droplet $\sigma$ evolution [11]

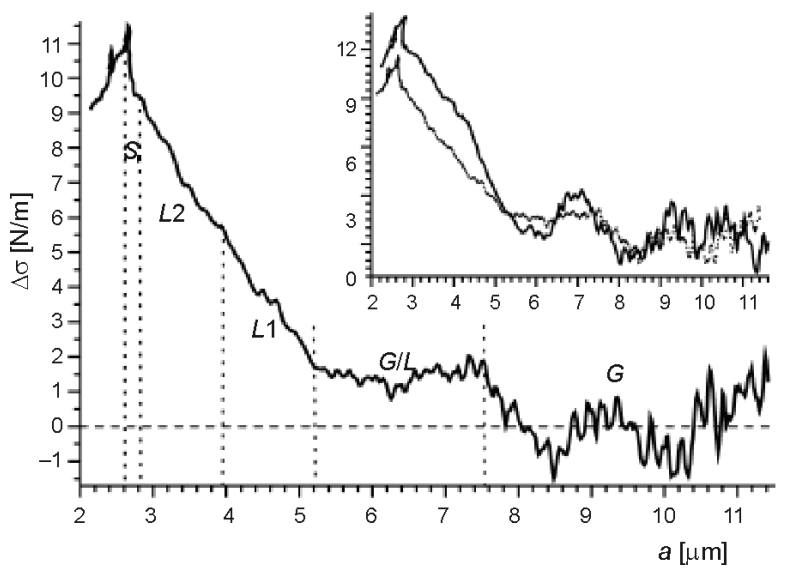

Fig. 18. Examples of surface pressure isotherms for droplets of suspensions of $200 \mathrm{~nm}$ polystyrene spheres. Phase transitions are marked with dotted lines

$$
a \dot{a}=\Lambda D_{a}\left(a, T_{a}\right)\left[\frac{p_{c c}\left(T_{c c}\right)}{T_{c c}}-\frac{p_{s}\left(T_{a}\right)}{T_{a}} \exp \left(\frac{\Lambda}{T_{a}} \frac{2 \sigma}{a}\right)\right]
$$

where $D$ is diffusion constant, $\mathrm{p}$ are pressures in climatic chamber $(c c)$ and saturated vapour at given temperature $T$, (a) describes values at the droplet surface, $\Lambda=M /(R F), M$ and $F$ are the molecular weight and density of the liquid, respectively, and $R$ is the universal gas constant.

Several regions (separated with vertical dashed lines in Fig. 18) can be distinguished in $\Delta \sigma(a)$ dependence. We associate these regions with various thermodynamic phases of the surface layer of inclusions $[12,13]$.

For $a>7.5 \mu \mathrm{m}$, there is a surface gas of inclusions on the droplet surface. Since in this region the experimental noise got amplified due to data processing, the modulation of the isotherm for $a>8.5 \mu \mathrm{m}$ cannot be unambiguously interpreted.

For $5.3>a>4 \mu \mathrm{m}$ we expect the liquid-expanded phase. The surface film can be described as a structure of fractal dimension below 2. The surface film is composed (as can be inferred from the simulation [12] of large loose aggregates. The evaporation of water leads to the compression of surface structures, as well as to the increase of the number of inclusions on the surface. As the fractal dimension of the surface structure increases, the film becomes denser.

For $a>4 \mu \mathrm{m}$ a phase transition to liquid-condensed (L2) is possibly observed. It manifests as the kink on the isotherm (best seen in Fig. 18).

Further compression leads to the surface liquid-solid transition for $a=2.7 \mu \mathrm{m}$. Then, after the rapid growth of the surface pressure, a collapse of the surface layer takes place.. The collapse followed by the formation of a multilayered surface solid manifests as the rapid decrease in the surface pressure.

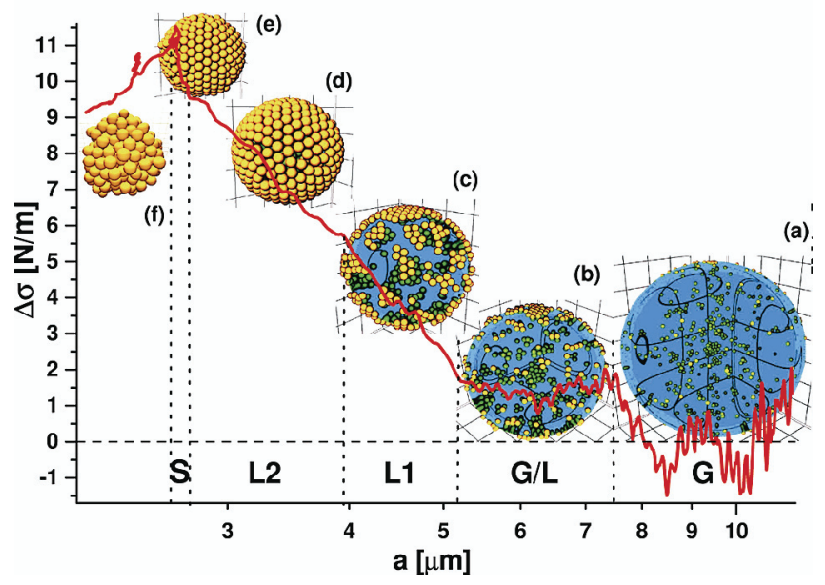

Fig. 19. Surface tension change semi logarithmic scale (red line) together with snapshots from a visualization of simulation presented in [12]. The droplet size has been scaled freely for best clarity 
To conclude:

The process of forming nanoparticle aggregates offers new possibilities of producing high-coverage spherical shells of nano- or microspheres. The spherical symmetry gives a unique opportunity for producing microcrystals of long-range ordering unaffected by the flatness of a substrate. The aggregation processes can be controlled by the thermodynamic parameters of the environment and the properties of nanospheres.

\section{Acknowledgments}

Finally, let me express my deep gratitude to all my coworkers: prof Krystyna Kolwas, dr Anastasjia Derkachova, dr Gennadji Derkachov,dr Daniel Jakubczy, dr Marcin Zientara, phd students Tho Duc and Mykola Schopa and Sławomir Palesa (technical staff)

The data and phenomena presented here are the results of their work.

\section{References}

[1] M. Faraday, Philos. Trans. R. Soc. London, 147, 145 (1857).

[2] Gustav Mie An.Phys. 25, 3777 (1908)

[3] http://en.wikipedia.org/wiki/Mie_theory

[4] http://en.wikipedia.org/wiki/Maxwell\%27s_equations

[5] K. Kolwas, Appl. Phys. B 66467 (1998),

[6] A. Derkachova, K. Kolwas, Eur. J. Phys. ST, 144, 93 (2007);

[7] K. Kolwas, S. Demianiuk, M. Kolwas, J. Chem. Phys. 106, 8436 (1997)

[8] D. Jakubczyk, M. Zientara, W. Bazhan, K. Kolwas, M. Kolwas, Opto-Electro. ReV. 9, 423 (2001).

[9] M. Zientara, D. Jakubczyk, K. Kolwas, M. Kolwas, J. Phys. Chem. A 112, 5152 (2008).

[10] Y. Li, P. Davidovits, Q. Shi, J. Jayne, C. Kolb, Worsnop, D. J. Phys. Chem. A, 105, 10627 (2001)

[11] H. Pruppacher, J. Klett, Microphysics of Clouds and Precipitation. Kluwer: Dordrecht, The Netherlands, 1997.

[12] G. Derkachov, K. Kolwas, D. Jakubczyk, M. Zientara, M. Kolwas, J. Phys. Chem. C, 112, 16919 (2008)

[13] D. Jakubczyk, M. Kolwas, G. Derkachov, K. Kolwas, J. Phys. Chem. C, 113 (24) 10598 (2009).

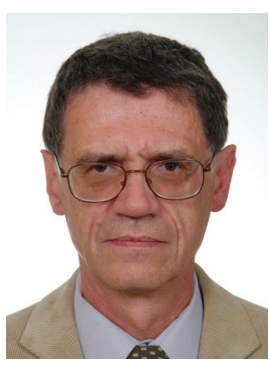

MACiEJ Kolwas, Professor (Ph.D. 1975, Dr. Habil. 1983), in years 1986-1992 Associate Professor and then Professor at the Institute of Physics of the Polish Academy of Sciences in Warsaw, Head of the Department of Physics of Radiation and Spectroscopy of the Institute of Physics 1985-1997, Head of Laboratory of Laser Spectroscopy since 1991, Deputy Director for Scientific Research of the Institute of Physics of the Polish Academy of Sciences 1997-1999, Head of the Postgraduate Studies at the Institute of Physics of the Polish Academy of Sciences since 1997-2009, Rector of Postgraduate Study of the Polish Academy of Sciences since 2000, General Secretary of the Polish Physical Society (1997-2001), President of the Polish Physical Society 2001-2005, President of the European Physical Society 2009-2011. Prof. M. Kolwas specialises in laser optics. 